\title{
Sobrepeso e obesidade em portadores do transtorno do espectro autista (TEA)
}

\section{Overweight and obesity in patients with autism spectrum disorder (ASD)}

\author{
1 Bruno Rocha Silva Setta brunosetta@hotmail.com \\ 1 Mayra Rozália Loureiro Novaes \\ 2 Lucrécia Helena Loureiro \\ 3 Márcia Dorcelina Trindade Cardoso \\ 1 Rider Santiago Alcoba Júnior
}

\footnotetext{
Discente do Curso de Medicina do Centro Universitário de Volta Redonda (UniFOA).

Coordenadora do Curso de Graduação em Enfermagem do Centro Universitário de Volta Redonda- UniFOA.

Docente do Curso de Graduação em Medicina do Centro Universitário de Volta Redonda- UniFOA.
}

\section{Resumo}

A frequência de sobrepeso e obesidade aumenta exponencialmente a cada ano no mundo todo, tornando-se uma comorbidade altamente prevalente em crianças e adolescentes, sobretudo em países desenvolvidos e emergentes. Diversos estudos têm demonstrado uma importante associação entre a prevalência dessas comorbidades em portadores do transtorno do espectro autista (TEA), no entanto poucos estudos têm se direcionado a crianças e adolescentes portadores desse transtorno. 0 objetivo deste artigo é avaliar os principais estudos realizados no período de 2009 a 2019, em relação à frequência de sobrepeso e obesidade nesse público, no Brasil. Para tanto, foi realizada uma revisão bibliográfica por meio da busca de artigos na Biblioteca Virtual em Saúde (BVS), PubMed e Google Acadêmico. Os descritores utilizados foram "sobrepeso", "obesidade" e "autismo". Verificou-se 11 artigos voltados estritamente para o público com TEA, mas apenas 5 analisaram a frequência de sobrepeso e obesidade. Existem muitas inconsistências metodológicas, especialmente no uso do IMC, na amostragem com poucos indivíduos e há a necessidade de incluir grupos de controle nas análises. 0 total de crianças e adolescentes diagnosticados com autismo em todos os artigos foi de 237 indivíduos. A maioria deles tinha entre 3 e 12 anos e era do sexo masculino. Dessa forma, foi possível observar a necessidade de realização de mais estudos e pesquisas direcionados à temática no país, associados também a atuações voltadas para a promoção de saúde e políticas públicas.

\section{Palavras-chave:}

Autismo. Sobrepeso. Obesidade.

\begin{abstract}
The frequency of overweight and obesity increases exponentially each year worldwide, making it a highly prevalent comorbidity in children and adolescents, especially in developed and emerging countries. Several studies have demonstrated an important association between the prevalence of overweight and obesity in patients with autism spectrum disorder (ASD), however, few studies have been addressed to children and adolescents with this disorder. The purpose of this article is to assess the main studies that were carried out in the period from 2009 to 2019, regarding to the frequency of overweight and obesity in this public in Brazil. Therefore, a bibliographic review was carried out by searching for articles in the Virtual Health Library (VHL), PubMed and Google Scholar. The keywords used were "overweight", "obesity" and "autism". There were found 11 articles aimed strictly at the public with TEA, but only 5 analyzed the frequency of overweight and obesity. There are many methodological inconsistencies, especially in the use of BMI, sampling with few individuals and the need to include control groups in the analyzes. Further, the total of children and adolescents diagnosed with autism in all articles were 237, most of them were between 3 and 12 years old and male. Thus, it was possible to observe the need to carry out more studies and research aimed at the theme presented in the country, also associated with health promotion actions and public policies.
\end{abstract}

\section{Keywords:}

Autism. Overweight. Obesity.

Como você deve citar?

SETTA, Bruno Rocha Silva et al. Sobrepeso e obesidade em portadores do transtorno do espectro autista (TEA). Cadernos UniFOA, Volta Redonda (RJ), v. 16, n. 46, p. 1-9 ago, 2021. 


\section{INTRODUÇÃO}

O autismo tem se destacado como uma importante temática, tanto em âmbito nacional quanto no internacional. Há uma ampla discussão, a partir de diferentes posições sobre os possíveis fatores etiológicos, da descrição de metodologias supostamente eficazes de tratamento, além da organização de políticas de cuidado e de dispositivos legais de garantia de direitos (OLIVEIRA et al., 2017).

A prevalência do autismo é estimada em 11,3 a cada 1.000 pessoas, sendo quase cinco vezes mais comum em meninos do que em meninas (CDC, 2012). Os diagnósticos de Transtorno do Espectro Autista (TEA) têm aumentado significativamente desde a década de 1960, e o último estudo relata que quase 1 em 68 crianças com 8 anos nos Estados Unidos já cumpriram os critérios para TEA, em 2010 (CDC, 2010).

No Brasil, não há estudos de prevalência de autismo em números oficiais, apenas há estimativas (JUNIOR, 2019). Contudo, há um Projeto de Lei da Câmara (PLC) dos Deputados, $n^{\circ}$ 139/18, que altera a Lei $n^{\circ} 7.853$, de 24 de outubro de 1989, para incluir especificidades inerentes ao autismo nos censos demográficos do Instituto Brasileiro de Geografia e Estatística (IBGE) (BRASIL, 2019). Estima-se que, no país, haja cerca de 2 milhões de autistas (OLIVEIRA, 2019).

Diante dos problemas comportamentais que desempenham um papel importante nos hábitos alimentares de crianças e adolescentes com TEA, seus padrões alimentares tendem a ser governados por aversão / recusa de alimentos ou preferências por certos tipos de alimentos às custas de outros (MARÍ-BAUSET et al., 2015). Além disso, os autistas dão preferência a alimentos mais densos em energia, têm aversão a certas texturas, cores e cheiros e ainda são recompensados com o alimento preferido, quando satisfazem ganhos comportamentais (SCHRECK et al., 2006; EVANS et al., 2012).

Dentre alguns problemas associados a esses aspectos, podemos destacar a presença do sobrepeso e da obesidade nessa população. Alguns estudos indicam que crianças com TEA têm 40\% mais chances que sejam obesos em comparação com crianças sem TEA (CURTIN et al., 2010). No entanto, evidencia-se uma carência de estudos sobre o estado nutricional de crianças e adolescentes com TEA no Brasil (KUMMER et al., 2015).

Em virtude da natureza complexa do TEA e considerando a relevância de se avaliar seu estado nutricional e possíveis comorbidades associadas, tais fatores constituem uma importante ferramenta de diagnóstico e prevenção de agravos à saúde dessa população, os quais possibilitam a implementação de estratégias multidisciplinares específicas à atenção integral da saúde. Dessa forma, o objetivo desta pesquisa foi avaliar, por meio de uma revisão bibliográfica, os estudos publicados no âmbito nacional, sobre a frequência de sobrepeso e obesidade em crianças e/ou adolescentes autistas.

\section{MÉTODO}

Esta revisão baseou-se em uma análise sistemática de estudos publicados no âmbito nacional, em relação à frequência de sobrepeso e obesidade em crianças e adolescentes diagnosticados com TEA.

Em cada estudo, foram identificados o autor e ano do estudo, o método de intervenção, a amostragem analisada e as suas características, objetivos do estudo e os resultados obtidos. 


\subsection{Procedimento de pesquisa}

Esta pesquisa foi realizada utilizando-se como bases de dados a Biblioteca Virtual em Saúde (BVS), PubMed e o Google Acadêmico, contemplando artigos nacionais publicados no período de 2009 a 2019. A escolha dessas plataformas se deve ao fato de elas contemplarem relevantes bancos de dados da área da saúde, tais como: Literatura Latino-americana e do Caribe em Ciências da Saúde (Lilacs), National Library of Medicine's (Medline) e Scientific Eletronic Library Online (Scielo), dentre outros.

Os descritores utilizados foram: "sobrepeso", "obesidade" e "autismo".

\subsection{Critérios de inclusão}

Como critérios adotados para esta revisão, os estudos teriam que ser originais e incluir pelo menos dois critérios: 1) os participantes deveriam ser crianças ou adolescentes com o diagnóstico de Transtorno do Espectro do Autismo (TEA); 2) os métodos de intervenção adotados deveriam se relacionar com a avaliação da frequência de sobrepeso e obesidade.

\subsection{Estudos selecionados}

Dos 5 estudos selecionados, apenas um trabalho avaliou a frequência de sobrepeso e obesidade, tanto em participantes diagnosticados com TDAH (Transtorno do déficit de atenção/hiperatividade) quanto diagnosticados com Perturbações do Espectro do Autismo de acordo com os critérios da DSMIV (AMERICAN PSYCHIATRIC ASSOCIATION, 2000). Além disso, apenas esse mesmo estudo envolveu a inclusão de um grupo-controle.

O número de artigos que avaliou apenas crianças e/ou adolescentes com TEA foi de 4 . A contabilização total dos participantes foi de 237 indivíduos, entre os 3 e 12 anos, com o maior predomínio do sexo masculino.

\subsection{Métodos de intervenção}

Os métodos de intervenção realizados foram: medidas antropométricas, como o peso e a altura (7), a circunferência do braço e as dobras cutâneas tricipital e subescapular (1) e circunferência da cintura (2), com posterior cálculo do índice de massa corporal (IMC) (6) e os percentis e escores-z do IMC específicos para a idade e gênero de pacientes (2).

Além disso, os pais dos participantes com TEA também foram avaliados em relação aos parâmetros antropométricos (1), com aplicação de questionários sociodemográficos (4), clínicos (1) e do modelo multinível bayesiano (1).

\section{RESULTADOS}

Com o levantamento realizado no Google Acadêmico e na BVS, foram encontrados apenas 5 artigos relacionados com o objeto desta pesquisa. 
Figura 1 - Ferramentas para avaliar a frequência de sobrepeso e obesidade em crianças e/ou adolescentes com TEA.

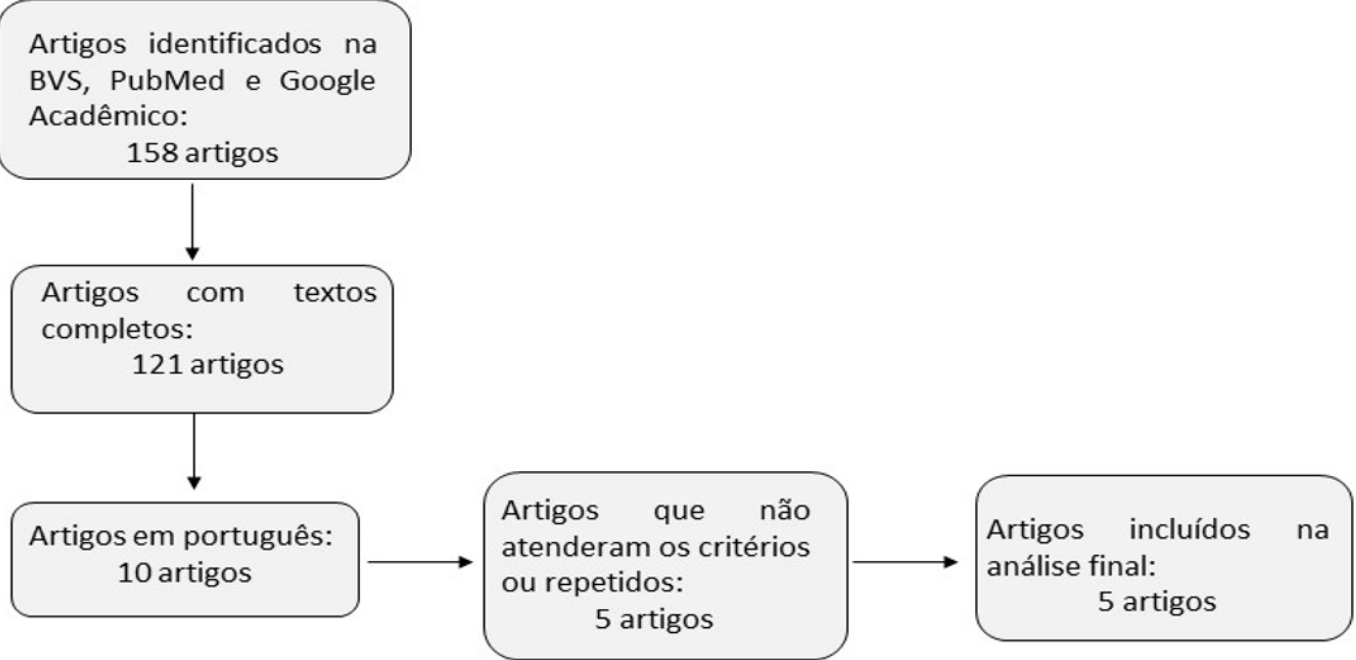

Fonte: Próprios autores. 
Quadro 1 - Resumo dos estudos realizados sobre a avaliação de sobrepeso e obesidade em crianças e/ou adolescentes com autismo.

\begin{tabular}{|c|c|c|c|c|}
\hline Autor/ano & Método de intervenção & Amostragem & Objetivos & Resultados obtidos \\
\hline $\begin{array}{l}\text { Toscano et al., } \\
2019\end{array}$ & $\begin{array}{l}\text { Estatura, massa corporal, } \\
\text { índice de massa corporal }\end{array}$ & $\begin{array}{l}88 \text { crianças entre } 3 \\
\text { e } 12 \text { anos }\end{array}$ & $\begin{array}{l}\text { Examinar o estado } \\
\text { de crescimento e } \\
\text { o desenvolvimento } \\
\text { físico de crianças com } \\
\text { TEA e a influência } \\
\text { de medicamentos } \\
\text { psicotrópicos }\end{array}$ & $\begin{array}{l}\text { Alta prevalência de } \\
\text { sobrepeso e obesidade } \\
\text { na primeira infância, } \\
\text { apesar de uma tendência } \\
\text { de redução substancial } \\
\text { na massa corporal e no } \\
\text { IMC ter sido aparente, } \\
\text { quando as crianças com } \\
\text { TEA entraram nos anos de } \\
\text { desenvolvimento púbere. }\end{array}$ \\
\hline $\begin{array}{l}\text { Domingues e } \\
\text { Szczerepa, } 2018\end{array}$ & $\begin{array}{l}\text { Questionário } \\
\text { sociodemográfico, } \\
\text { estatura e peso }\end{array}$ & $\begin{array}{l}25 \text { participantes } \\
\text { com idade } \\
\text { entre } 4 \text { a } 11 \text { anos }\end{array}$ & $\begin{array}{l}\text { Avaliar o perfil } \\
\text { nutricional e os } \\
\text { hábitos alimentares de } \\
\text { crianças portadoras do } \\
\text { TEA }\end{array}$ & $\begin{array}{l}64 \% \text { eram obesos, } 24 \% \\
\text { eram eutróficos e } 12 \% \\
\text { apresentavam magreza. }\end{array}$ \\
\hline $\begin{array}{l}\text { Caetano e Gurgel, } \\
2018\end{array}$ & $\begin{array}{l}\text { Questionário } \\
\text { sociodemográfico, } \\
\text { histórico nutricional, } \\
\text { estatura, peso, } \\
\text { circunferência do braço } \\
\text { e as dobras cutâneas } \\
\text { tricipital e subescapular } \\
\text { e IMC } \\
\end{array}$ & $\begin{array}{l}26 \text { crianças, entre } 3 \\
\text { e } 10 \text { anos de idade }\end{array}$ & $\begin{array}{l}\text { Avaliar o estado } \\
\text { nutricional e o } \\
\text { consumo alimentar de } \\
\text { crianças portadoras do } \\
\text { transtorno do espectro } \\
\text { autista (TEA) }\end{array}$ & $\begin{array}{l}\text { Magreza: } 3,85 \%(n=1), \\
\text { eutrofia } 19,23 \%(n=5), \\
\text { risco de sobrepeso } 38,46 \% \\
(n=10), \text { sobrepeso } 23,08 \% \\
(n=6) \text { e obesidade } 15,38 \% \\
(n=4) .\end{array}$ \\
\hline $\begin{array}{l}\text { Almeida et al., } \\
2018\end{array}$ & $\begin{array}{l}\text { Questionário } \\
\text { sociodemográfico, } \\
\text { estatura, peso, IMC }\end{array}$ & $\begin{array}{l}29 \text { crianças entre } 3 \\
\text { e } 12 \text { anos }\end{array}$ & $\begin{array}{l}\text { Analisar o consumo } \\
\text { de alimentos } \\
\text { ultraprocessados entre } \\
\text { crianças com TEA e sua } \\
\text { associação ao estado } \\
\text { nutricional }\end{array}$ & $\begin{array}{l}20,7 \%(n=6) \text { eram obesos, } \\
34,5 \%(n=10) \text { tinham } \\
\text { sobrepeso, } 24,1 \%(n=7) \\
\text { eram eutróficos e } 20,7 \%(n \\
=6) \text { tinham baixo peso }\end{array}$ \\
\hline $\begin{array}{l}\text { Kummer et al., } \\
2015\end{array}$ & $\begin{array}{l}\text { Questionário } \\
\text { sociodemográfico e } \\
\text { clínico, peso, estatura, } \\
\text { circunferência abdominal } \\
\text { e IMC }\end{array}$ & $\begin{array}{l}69 \text { crianças e } \\
\text { adolescentes, entre } \\
4 \text { e } 8 \text { anos de idade }\end{array}$ & $\begin{array}{l}\text { Avaliar a frequência } \\
\text { de sobrepeso e } \\
\text { obesidade em crianças } \\
\text { e adolescentes com } \\
\text { transtorno do espectro } \\
\text { do autismo (TEA) e } \\
\text { transtorno do déficit de } \\
\text { atenção/hiperatividade } \\
\text { (TDAH) e em seus } \\
\text { pais, em comparação } \\
\text { com crianças e } \\
\text { adolescentes da } \\
\text { comunidade sem } \\
\text { transtornos do } \\
\text { desenvolvimento. }\end{array}$ & $\begin{array}{l}\text { Crianças e adolescentes } \\
\text { com TEA e TDAH exibiram } \\
\text { maior percentil }(p<0,01) \text { e } \\
\text { escore-z }(p<0,01) \text { do IMC } \\
\text { em relação aos controles, } \\
\text { bem como frequência } \\
\text { mais elevada de sobrepeso } \\
\text { e obesidade ( } p=0,04) \text {. } \\
\text { Os pacientes com TEA e } \\
\text { TDHA não diferiram entre } \\
\text { si quanto a essas variáveis } \\
\text { ou quanto à circunferência } \\
\text { abdominal. }\end{array}$ \\
\hline
\end{tabular}

Fonte: Próprios autores.

\section{DISCUSSÃO}

Apenas em 27 de dezembro de 2012, foi sancionada a Lei n¹2.764, que "Institui a Política Nacional de Proteção dos Direitos da Pessoa com Transtorno do Espectro Autista" (BRASIL, 2012). Por essa lei, também se reconhece o indivíduo com TEA como "pessoa com deficiência, para todos os efeitos legais". A partir desse marco importante para o público-alvo desta pesquisa, pode-se verificar que todos os estudos identificados (5) foram publicados após o ano de 2012. 
O sobrepeso e a obesidade são problemas de saúde pública que propiciam o desenvolvimento de doenças crônicas na vida adulta. Esses problemas podem aumentar o risco na criança e/ou adolescente de desenvolver o diabetes, doenças cardiovasculares e psicossociais (KUMMER et al., 2015).

Em relação à amostragem utilizada nos diversos estudos, é possível verificar que houve, de um modo geral, a presença de pequenos grupos, com a média de 29 participantes. Contudo, em alguns casos, apenas um interveniente estava presente. Majoritariamente, são crianças ou adolescentes diagnosticados com autismo, entre 3 e 12 anos de idade, e são do sexo masculino.

Diante dos estudos demonstrados, os autores pretendiam avaliar o estado nutricional de crianças e/ou adolescentes de diversas formas: questionários sociodemográficos, estatura, peso, IMC, percentil e circunferência abdominal. Vale ressaltar que poucos estudos (2) utilizaram o método da circunferência abdominal para essa finalidade. Trata-se de um método simples, de fácil execução e de baixo custo (PEIXOTO et al., 2006), embora não diferencie gordura intra-abdominal da subcutânea (SARNI et al., 2006).

A maioria dos estudos (5) utilizou o IMC como método principal para avaliar o estado nutricional. Esse método é calculado pela razão peso $(\mathrm{kg}) /$ estatura $(\mathrm{m})^{2}$ e é bastante utilizado, devido à facilidade manusear o seu cálculo. No entanto, ele não reflete a gordura corporal, tampouco sua distribuição, sendo importante correlacioná-lo com outras medidas independentes de composição corporal, como a massa de gordura corporal (MGC) ou o percentual de gordura corporal (\% GC) (KUMMER et al., 2015).

Apenas o trabalho de Kummer et al. (2015) utilizou um grupo-controle dentre os estudos analisados. Com a inclusão de um grupo-controle no trabalho desses autores, pode-se afirmar que a amostra de crianças e adolescentes com TEA analisada foi mais propensa a desenvolver sobrepeso e obesidade, quando comparados com a população geral.

No trabalho de Toscano et al. (2019), foram utilizados os dados específicos para a idade de referência para a população norte-americana, oriundos do CDC (Centers for Disease Control and Prevention), para comparar os dados de estatura, massa corporal e do peso, com base em valores de ponto de corte do IMC específicos para os dados de referência, assim como os valores de ponto de corte do IMC específicos praticados no Brasil. Os autores verificaram altos valores de IMC, principalmente entre quatro e oito anos, comparados com a referência específica para a idade de crianças normais e comparáveis também com valores de corte do IMC específicos para a população brasileira. No entanto, os próprios autores apontam as limitações do estudo, que considerou apenas meninos com TEA, uma vez que a amostra de meninas era muito limitada, e que o fato de considerar as referências americanas pode ser insensível para se aplicar em crianças e adolescentes no Brasil, devido à variabilidade étnica e socioeconômica.

Observamos que os outros quatro trabalhos também indicam alta frequência de sobrepeso e obesidade, mas com a ausência de um grupo-controle nas análises, o que restringe substancialmente a interpretação dos achados. Diferentemente dos demais estudos que utilizaram desvio-padrão e/ou coeficiente de variação, o trabalho de Toscano et al. (2019) utilizou o modelo bayesiano como método de análise estatística para descrever os padrões de crescimento individual.

Sobre os aspectos sociodemográficos, apenas os trabalhos de Almeida et al. (2018), Domingues e Szczerepa (2018) e Caetano e Gurgel (2018) informaram a média das rendas familiares dos participantes das suas pesquisas. As amostras recebiam entre 1 a 2 salários mínimos, mas vale ressaltar que, no trabalho de Almeida et al. (2018), a renda mensal foi estratificada em menor que 1 salário mínimo ou maior que 1 salário mínimo. 
Segundo Almeida et al. (2018), 72,5\% ( $n=21)$ dos responsáveis/pais entrevistados possuíam ensino médio incompleto ou completo. 0 trabalho de Caetano e Gurgel (2018) informa, na metodologia, que foi aplicado um questionário sociodemográfico contendo a escolaridade dos entrevistados, porém percebe-se que não houve a discussão dessa variável no trabalho. Nos demais trabalhos, os autores não incluíram essas duas variáveis.

0 tratamento e o cuidado dos portadores do TEA exigem, muitas vezes, terapias multiprofissionais rotineiramente (psicólogos, nutricionistas, fonoaudiólogos, entre outros), uso de medicamentos de controle dos distúrbios e sintomas relacionados à emoção, ansiedade, tensão, entre outros, e de uma nutrição adequada (KUMMER et al., 2015). Em relação a esse último aspecto, muitos autistas apresentam problemas gastrointestinais, como a intolerância ao glúten (proteína do trigo) ou à caseína (proteína do leite), por exemplo, exigindo uma alimentação restrita a esses nutrientes que ainda é cara (DOMINGUES E SZCZEREPA, 2018). Assim, a baixa renda familiar e escolaridade dos responsáveis pelos autistas podem impactar significativamente no estado nutricional deles.

Alguns medicamentos, como antipsicóticos (risperidona), estimulantes (metilfenidato), antidepressivos (fluoxetina) e/ou antiepiléticos (carbamazepina) podem estar presentes na rotina do portador do TEA e induzir ao ganho de peso. Condizente com a literatura médica, nos trabalhos de Kummer et al. (2015), Caetano e Gurgel (2018) e Toscano et al. (2019), houve uma tendência de apresentação de percentil mais alto do IMC nos participantes com TEA que faziam o uso de risperidona. Algumas alterações metabólicas, como o aumento da resistência à insulina, hiperglicemia, hipertensão arterial e dislipidemia, podem ser consequências do uso desse fármaco (GALLING e CORRELL, 2015), os quais podem justificar o ganho de peso.

Pôde-se perceber no trabalho de Kummer et al. (2015) que os pais das crianças com TEA não diferem em relação ao IMC, circunferência abdominal e frequência de sobrepeso/obesidade. No entanto, essa afirmação não está totalmente fidedigna, pois, de acordo com os autores, não foi possível avaliar os pais do grupo-controle, já que as crianças e adolescentes desse grupo foram avaliados na escola.

\section{CONSIDERAÇÕES FINAIS}

Evidencia-se o baixo número de trabalhos nacionais publicados em relação à avaliação de sobrepeso e obesidade em crianças e adolescentes portadores de TEA. Essa temática torna-se relevante e necessária, expondo benefícios nos diferentes domínios, sendo significativa a influência desses problemas de saúde pública em pessoas com autismo, seja ao nível da sua condição física, seja nas suas capacidades cognitivas e sensoriais.

Destaca-se a necessidade de não se utilizar o IMC como único método antropométrico para se avaliar o sobrepeso e obesidade em autistas, assim como a necessidade da inclusão de grupos-controle nos estudos, como forma de melhorar a interpretação dos achados da pesquisa.

O acompanhamento nutricional torna-se fundamental aos portadores de TEA para se minimizar o risco de deficiências nutricionais devido a sua seletividade nutricional, problemas gastrointestinais ou pelo uso de medicamentos psicóticos. Além disso, ressalta-se a importância de terapias multiprofissionais para que isso ocorra.

Dessa forma, é de extrema importância o desenvolvimento de novas pesquisas para melhorar a forma de abordagem profissional, de atuações voltadas para a promoção da saúde (prática de atividades 
físicas, culturais ou de lazer) e que profissionais de saúde realizem periodicamente a avaliação ponderal e orientem seus pacientes com TEA ou seus responsáveis sobre hábitos saudáveis de vida.

\section{REFERÊNCIAS}

ALMEIDA, A. K. A. et al. Consumo de ultraprocessados e estado nutricional de crianças com transtorno do espectro do autismo. Revista Brasileira em Promoção da Saúde, 31 (3): 1-10, jul./set., 2018.

American Psychiatric Association. Diagnostic and StatisticalManual of Mental Disorders. 4th ed. American Psychiatric Association; 15. 2000.

BRASIL. Projeto de Lei da Câmara ${ }^{\circ}$ 139, de 2018. Estabelece que os censos demográficos realizados a partir de 2018 incluirão as especificidades inerentes ao autismo. Disponível em: https://www25.senado. leg.br/web/atividade/materias/-/materia/134964. Acesso em: 11 out. 2019.

BRASIL. Lei n ${ }^{\circ}$ 10.764, de 27 de dezembro de 2012. Institui a Política Nacional de Proteção dos Direitos da Pessoa com Transtorno do Espectro Autista; e altera o $\S 3^{\circ}$ do art. 98 da Lei ${ }^{\circ} 8.112$, de 11 de dezembro de 1990. Diário Oficial [da] República Federativa do Brasil, Brasília, DF, 2012.

CAETANO, M. V.; GURGEL, D. C. Perfil nutricional de crianças portadoras do transtorno do espectro autista. Revista Brasileira em Promoção da Saúde, 31 (1): 1-11, jan./mar., 2018.

CDC. Centers for Disease Control and Prevention. 2012. Prevalence of autism spectrum disorders. Surveillance Summaries MMWR March 29. Disponível em: http://www.cdc.gov/ media/releases/2012/ a0329_autism_disorder.html. Acesso em: 24 maio. 2020.

CURTIN C.; ANDERSON, S. E.; MUST, A.; BANDINI, L. The prevalence of obesity in children with autism: a secondary data analysis using nationally representative data from the National Survey of Children's Health. BMC Pediatrics, p.10-11, 2010.

Developmental Disabilities Monitoring Network Surveillance Year 2010 Principal Investigators, Centers for Disease Control and Prevention (CDC). Prevalence of autism spectrum disorder among children aged 8 years - autism and developmental disabilities monitoring network, 11 sites, United States, 2010.

MorbMortal Wkly Rep Surveill Summ, 63:1- 21. 2014.

DOMINGUES, R. C. P.; SZCZEREPA, S. B. Avaliação nutricional de crianças portadoras do transtorno do espectro autista em uma instituição filantrópica de Ponta Grossa - PR. Revista Nutrir, 9a Ed. (jan - jul) de 2018.

EVANS, E. W. et al. Dietary patterns and body mass index in children with autism and typically developing children. Research in Autism Spectrum Disorders, 6(1), 399 - 405, 2012.

GALLING, B.; CORRELL, C. U. Do antipsychotics increase diabetes risk in children and adolescentes. Expert Opin Drug Saf. 2015;14(2):219 - 241.

JUNIOR, F. P. Quantos autistas há no Brasil? Disponível em: https://www.revistaautismo.com.br/noticias/ quantos-autistas-ha-no-brasil/. Acesso em: 12 out. 2019. 
KUMMER, A. et al. Frequência de sobrepeso e obesidade em crianças e adolescentes com autismo e transtorno do déficit de atenção/hiperatividade. Revista Paulista de Pediatria, 2016; 34(1):71-77.

MARÍ-BAUSET, S. et al. Anthropometric measures of Spanish children with autism spectrum disorder. Research in Autism Spectrum Disorders, v. 9, p. 26-33, 2015.

OLIVEIRA, B. D. C. et al. Políticas para o autismo no Brasil: entre a atenção psicossocial e a reabilitação. Revista de Saúde Coletiva, Rio de Janeiro, 27 [3]: 707-726, 2017.

OLIVEIRA, C. Um retrato do autismo no Brasil. Disponível em: http://www.usp.br/espacoaberto/?materia=umretrato-do-autismo-no-brasil. Acesso em: 11 out. 2019.

PEIXOTO, M. R. G. et al. Circunferência da cintura e índice de massa corporal como preditores da hipertensão arterial. Arquivos Brasileiros de Cardiologia, 87:462-70, 2006.

SARNI, R. S. et al. Relação da cintura abdominal com a condição nutricional, perfil lipídico e pressão arterial em pré-escolares de baixo estrato socioeconômico. Arquivos Brasileiros de Cardiologia, 87(2):153-8, 2006.

SCHRECK, K. A.; WILLIAMS, K. Food preferences and factors influencing food selectivity for children with autism spectrum disorders. Research in Developmental Disabilities, n. 27, p. 353-63, 2006.

TOSCANO, C. V. A. et al. Crescimento e massa corporal em crianças brasileiras com transtornos do espectro autista: um estudo longitudinal misto. Jornal de Pediatria, 95(6):705-712, 2019. 